\title{
Maximization of Feature Potential Mutual Information in Multimodality Image Registration Using Particle Swarm Optimization
}

\author{
Xuan YANG $^{* a}$, Jihong PEI ${ }^{\mathrm{b}}$, Weixin $\mathrm{XIE}^{\mathrm{a}}$ \\ ${ }^{\mathrm{a}}$ College of Information and Engineering, Shenzhen University, Guangdong, 518060, China \\ ${ }^{b}$ Modern Education Technology and Information Center, Shenzhen University, 518060, China
}

\begin{abstract}
Standard Mutual Information function contains local maxima, which make against to convergence of registration transformation parameters for automated multimodality image registration problems. We proposed Feature Potential Mutual Information (FPMI) to increases the smoothness of the registration measure function and use Particle Swarm Optimization to search the optimal registration transformation parameter in this paper. At first, Edges of images are detected. Next, edge feature potential is defined by expanding edges to the neighborhood region using potential function. Each edge point influences the whole potential field, just like the particle of physics in the gravitation field space. FPMI is computed on the edge feature potential of two images. It substitutes the edge feature potential values for gray values in images. It can avoid great change of joint probability distribution and has less local maxima. The registration accuracy of FPMI is analyzed under different edge detection cases. It is shown that the registration accuracy of FPMI is more accurate and more robust than that of MI. Maximization of FPMI is done by PSO. PSO combines local search methods with global search methods, attempting to balance exploration and exploitation. Its complex behavior follows from a few simple rules and has less computational complexity. Multimodal medical images are used to compare the response of FPMI and MI to translation and rotation. Experiments show that FPMI is smoother and has less local fluctuations than that of MI. Registration results show that PSO do it better than Powell's method to search the optimal registration parameters.
\end{abstract}

Keywords: Image Registration, Mutual Information, Particle Swarm Optimization

\section{INTRODUCTION}

In clinical applications multimodality medical images are frequently fused together to improve the diagnostic accuracy. Registration of multimodal medical images is an important first step in successful fusion of those images. Image registration is a procedure that determines the best match between multimodal images of the same object field.

Mutual information (MI) to describe the degree to which an image can be predicted from the other has been proposed by Viola et al. ${ }^{[6]}$ and Collignon ${ }^{[7]}$. If the two images are correctly matched, knowing one image gives information about the other. Therefore their mutual information is high. Conversely, two independent images will have very low mutual information. The mutual information between two images has been shown to align images from different modalities accurately and robustly ${ }^{[1,5-8,10-12]}$. However, the local maxima of mutual information make it difficult to register images, because the search algorithm will converge to the local maximum easily ${ }^{[2-4,9]}$. Ji et al. ${ }^{[2]}$ analyzed both sampling and interpolation effects of mutual information. He discussed several preprocessing methods for reducing the interpolation effects also. Pluim ${ }^{[3]}$ analyzes the artifacts of linear interpolation and partial volume interpolation methods in the mutual information. He has furthermore shown that a small resampling of one of the images vastly increases the smoothness of the mutual information. Likar and Pernus ${ }^{[8]}$ proposed a combination of prior and floating information on the joint probability and random re-sampling of one image to improve the registration. Tsao ${ }^{[4]}$ analyzed artifacts from eight interpolators and proposed several strategies to reduce artifacts and improve registration robustness. Pluim ${ }^{[5]}$ combined both standard mutual information and gradient information to yield a better registration.

Mutual information does not contain spatial information, but spatial information is of great benefit to registration of multimodal images. In this paper, a new method to compute mutual information between two images, which is not based on intensity, but is based on spatial information of images, is proposed to register multimodal medical images. Edges of images are detected by using canny operator at first. Then feature potential of image is defined by expanding

xyang0520@263.net; 
the edge to the neighborhood region using potential function based on the thought of physics field. Feature potential mutual information is computed by the feature potential of two images. Analysis and experiments show that FPMI function has less local maxima, and is suitable to different interpolation methods. Finally, particle swarm optimization is used to search the optimal registration transformation parameter. Analysis and experiments show that our method is more accurate and more robust to register multimodal medical images than maximization of standard mutual information.

\section{FEATURE POTENTIAL MUTUAL INFORMATION}

\subsection{Mutual Information}

Mutual information is a basic concept from information theory, measuring the statistical dependence between two random variables or the amount of information that one variable contains about the other. The mutual information $I$ of two images $X$ and $Y$ is evaluated as ${ }^{[6]}$ :

$$
\begin{aligned}
& I(X, Y)=H(X)+H(Y)-H(X, Y) \\
& H(X, Y)=-\sum_{x, y} P(x, y) \log P(x, y)
\end{aligned}
$$

where $H(X, Y)$ is the Shannon entropy of the joint entropy, computed on the joint probability distribution of the intensity values. $H(X)$ and $H(Y)$ are entropy of image $X$ and $Y$, computed on the marginal probability distributions $P(x)$ and $P(y)$ alternatively. Mutual information will be at its maximum when the images matched. In order to be less sensitive to the changes in overlap, Studholme ${ }^{[11]}$ proposed normalized mutual information and Maes ${ }^{[12]}$ defined entropy correlation coefficient (ECC) also.

The mutual information function could contain local maxima, when the images are of low resolution, when the images contain little information, or when interpolation images with different resolution are used. The disadvantage impedes the registration optimization process and rule out the subpixel accuracy.

\subsection{Feature Potential Mutual Information}

Ji et al. ${ }^{[2]}$ showed that local fluctuations of MI can be caused by intensity variation of images. In order to reduce the influence of intensity variation of images, we proposed a new method to compute mutual information between two images in this paper, which is not based on intensity, but is based on spatial information of images, which is called feature potential mutual information.

At first, edges of images are detected by using canny operator. In order to reduce the sensitivity of edge detection results to noise and to the parameters of detection operator, we expand the edge to the neighborhood region using potential function. The thought of field theory of physics is applies to define edge feature potential. Each edge point $(x, y)$ influences the whole potential field, just like the particle of physics in the gravitation field space. Based on this thought, we define the feature potential, which map the edges to feature potential field. At first, potential function $P(x, y)$ is defined as two dimensional gauss function,

$$
P(x, y)=\frac{1}{2 \pi \sigma^{2}} \exp \left(-\frac{x^{2}+y^{2}}{2 \sigma^{2}}\right)
$$

The feature potential function $P_{E}(x, y)$ is defined as,

$$
P_{E}(x, y)= \begin{cases}\sum_{(u, v) \in R} P(x-u, y-v) & (x, y) \in R \\ 0 & (x, y) \notin R\end{cases}
$$

where $R$ is the set of edge points of image. $\sigma$ can be used to adjust the range of feature potential function. The definition of feature potential function is based on the principle, "the closer the points are, the more influence between them they have".

Feature potential mutual information is computed on the feature potential function of two images. The joint probability $P_{F P}(x, y)$ of feature potential mutual information is,

$$
P_{F P}(x, y)=\frac{N(x, y)}{N_{F}}
$$


where $N(x, y)$ is the number of edge feature potential pair $\left(P_{E 1}(i, j), P_{E 2}(i, j)\right), \quad x=P_{E 1}(i, j), \quad y=P_{E 2}(i, j)$ of two images. $\quad N_{F}$ is the total number of edge feature potential pair. The margin probability function is

$$
P_{F P}(x)=\sum_{y} P_{F P}(x, y) \quad P_{F P}(y)=\sum_{x} P_{F P}(x, y)
$$

Feature potential function merged small edges together and spread the major spatial structure of images from lines to regions. Little difference of the edge detection results would not result in distinct difference of FPMI. FPMI is robust to noise and is insensitive to the parameters of edge detection operators. Because feature potential function is smooth, FPMI computed with the feature potential of two images would have less local fluctuations. Moreover, Feature potential illustrates the spatial structure of images. So FPMI could be used as a registration measure to register multimodality images. This thought is just like the registration method based on image segmentation, but it is different from these methods because feature potential is a feature based on regions not based on lines. FPMI is robust to noise and is insensitive to the parameters of edge detection operators. So it overcomes the disadvantage of the registration method based on image segmentation.

\section{$2.3 \sigma$ of potential function}

Feature potential mutual information can be adjusted with the $\sigma$ of potential function. Figure 1 is the feature potential of an image with different $\sigma$. We can see that the feature potential represents the space structure of objects. Feature potential is not sensitive to the edge detection result, that is, although edge detection results of image are different, the feature potential is similar. This characteristic is very well and could overcome the disadvantage of registration method, which is based on image edges only.
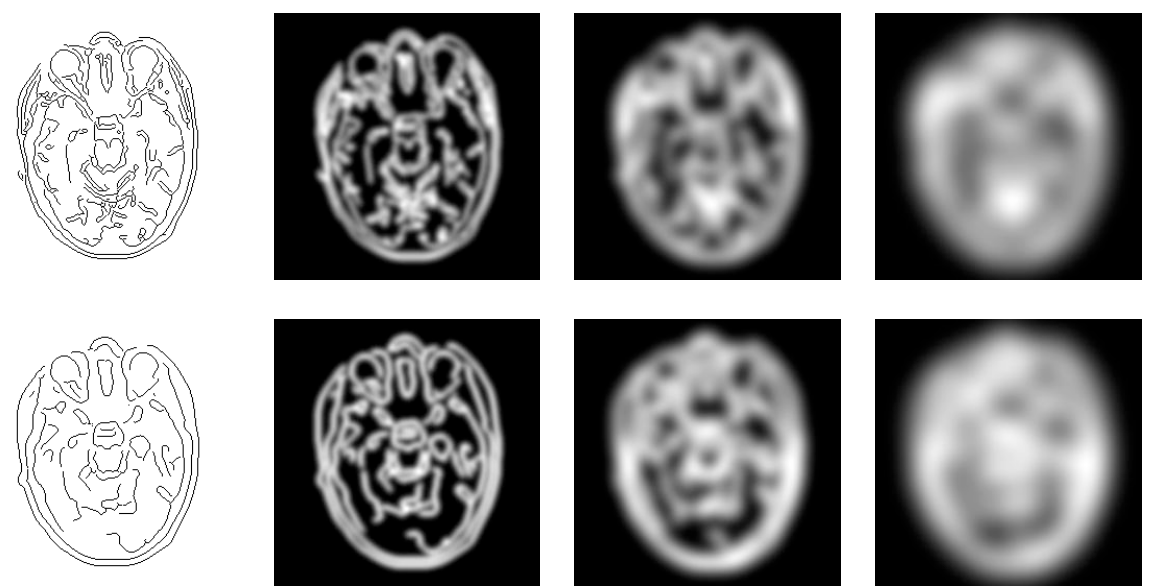

Figure 1: the first column is edges of an image detected by canny operator with two different scales. The second column is the feature potential corresponding left edge results with $\sigma=3.0$. The third column is the feature potential with $\sigma=7.0$. The fourth column is the feature potential with $\sigma=13.0$.

Feature Potential is related to $\sigma$ of potential function. The value of $\sigma$ could not be too large or too small. If $\sigma$ is too large, feature potential is merged too severely to distinguish, which make against to register images, such as the third column of figure 1. If $\sigma$ is too small, feature potential is so sharp that it just like edges of image, which could not be used to compute mutual information, such as the first column of figure 1. The value of $\sigma$ is adjusted based on the distance of between edges.

(1) If the distance between two parallel close adjacent edges is $d$, their feature potential should be expanded to form one potential apex between them to avoid the local fluctuations of FPMI. It can be deduced that the potential value of the point locating in the middle of two edges along the edge direction is (in order to simplify analysis, only two points along edge direction is considered),

$$
P(\sigma)=\frac{\sqrt{2}}{\sqrt{\pi} \sigma} \exp \left(-\frac{d^{2}}{8 \sigma^{2}}\right)
$$

When the fellow formula satisfied, a potential apex would appear in the middle of two edges, 


$$
P(\sigma)=\frac{\sqrt{2}}{\sqrt{\pi} \sigma} \exp \left(-\frac{d^{2}}{8 \sigma^{2}}\right)>\frac{1}{\sqrt{2 \pi} \sigma}
$$

That is $\sigma>0.424 d$. It means, in order to avoid the local fluctuations of feature potential mutual information, the maximum potential should be in the middle of two close adjacent edges. The corresponding $\sigma$ should be $0.424 d$ at least.

(2) If two edges are the major structure of images, they could not be merged together. $\sigma$ could not be too large. Suppose the distance of two edges is $d$, in order to separate their feature potential function, $\sigma<d / 6 \sqrt{2}$ should be satisfied.

The value range of $\sigma$ can be estimated with the distance of edges. Any value of $\sigma$ in this range is appropriate. In generally, we choose the big one in this range to avoid the local fluctuations of feature potential mutual information function.

\section{REGISTRATION ACCURACY OF FPMI}

We analyze the registration accuracy of FPMI under different edge detection cases.

\subsection{Registration of single Edges with same width}

In order to simplify analysis, one-dimension discrete signals are used to illustrate the registration accuracy of FPMI. Let $X$ and $Y$ be single slope edges with same width, which are used to do registration. Suppose $X$ and $Y$ are central symmetry. Edges detected by Canny operator are located in the middle of two singles. The corresponding feature potentials are two gauss function with same width (because $\sigma$ of potential function is same) as figure 2 .

Suppose the length of each sample point is 1 . The length of $X$ and $Y$ is $L$, which is the length of potential feature signal also. Suppose the finite length of gauss function is $M$. We translate signal $\mathrm{Y}$ along horizon direction.

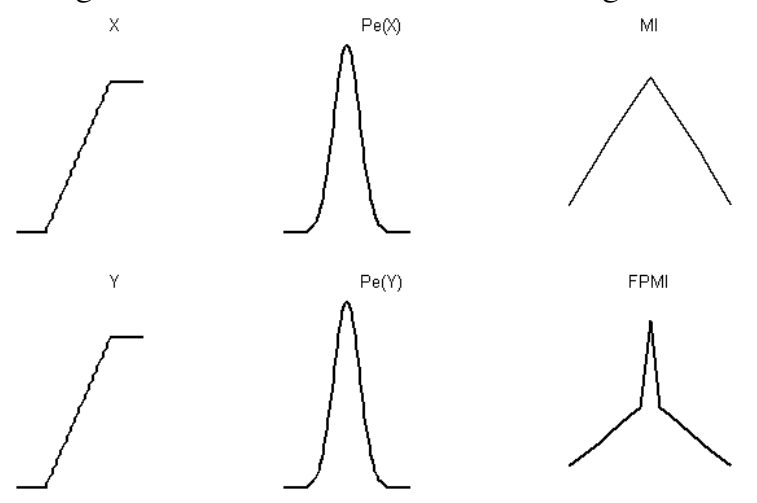

Figure 2: the first column is two edges with same edge width. The second column is the feature potential corresponding left edge results. The up item of the third column is response of mutual information to horizon translation; the down item of the third column is the response of the FPMI to horizon translation.

When signal Y shifts $x$ rightwards and $x \leq M$. It can be deduced that the FPMI is

$$
F P M I_{x}=-\frac{L-M-2 x}{2 L-2 x} \log \frac{L-M-2 x}{2 L-2 x}-\frac{L-M}{L-x} \log \frac{L-M}{2 L-2 x}-\frac{M-x}{L-x} \log \frac{1}{L-x}
$$

Suppose $x<L-M$, above formula can be approximated as,

$$
\begin{aligned}
F P M I_{x} & \approx-\frac{L-M-2 x}{2 L-2 x} \log \frac{L-M}{2 L}-\frac{L-M}{L-x} \log \frac{L-M}{2 L}-\frac{M-x}{L-x} \log \frac{1}{L} \\
& =-\log \frac{L-M}{2 L}-\left(1-\frac{L+M}{2 L-2 x}\right) \log \frac{L-M}{2 L}+\left(1-\frac{L-M}{L-x}\right) \log \frac{L-M}{2}
\end{aligned}
$$

Apparently, $F P M I_{x}$ is a monotonic decrease function with $x$. Similarly, when signal $\mathrm{Y}$ shift $x$ leftwards, $F P M I_{x}$ is a monotonic decrease function with $x$ also. That means a maximum occurs at $x=0$. The conclusion is that local maxima of FPMI would not appear. It is same to standard mutual information. That is to say, when MI does 
registration well, FPMI could do registration well also. Because the location of single edge detected by Canny operator is accurate, the registration result of FPMI is accurate also.

\subsection{Registration of single Edges with different width}

Let $X$ and $Y$ be single edges with different width. Edges detected by Canny operator are located in the middle of two singles. Because we use the same $\sigma$ to compute feature potential, so the corresponding feature potentials are two gauss singles with same width as figure 3. It is same to 3.1 that FPMI is maximum when two signals are matched, and FPMI decrease monotonously when $Y$ shifts rightwards or leftwards. That is to say, local maxima of FPMI would not appear when the two edges width is different.

However, mutual information will be minimum when two edges with different width registered. Let the length of signal is $L$, where the slop length of $X$ is $M$, and the slop length of $Y$ is $N$. Suppose $N<M$. Suppose two signals are central symmetry. When the center points of the two signals matched, two signals matched.

Signal $Y$ shifts $x$ rightwards. It satisfies $x \leq \frac{M-N}{2}$. That means the slop of $Y$ is in the range of the slop of $X$. The mutual information is

$$
M I_{x}=-\frac{L-N-2 x}{2 L-2 x} \log \frac{L-N-2 x}{2 L-2 x}-\frac{L-N}{2 L-2 x} \log \frac{L-N}{2 L-2 x}-\frac{N}{L-x} \log \frac{1}{L-x}
$$

Suppose $x<L-N$, formula (11) can be approximated as,

$$
\begin{aligned}
M I_{x} & \approx-\frac{L-N-2 x}{2 L-2 x} \log \frac{L-N}{2 L}-\frac{L-N}{2 L-2 x} \log \frac{L-N}{2 L}-\frac{N}{L-x} \log \frac{1}{L} \\
& =-\log \frac{L-N}{2 L}+\frac{N}{L-x} \log \frac{L-N}{2}
\end{aligned}
$$

Apparently, $M I_{x}$ is a monotonic increase function with $x$. Similarly, when signal Y shift $x$ leftwards, $M I_{x}$ is a monotonic increase function with $x$ also. That means a local minimum occurs at $x=0$. We can prove the same case when $M<N$. The conclusion is that, when edges width is different, the response of mutual information to horizon translation would have local minimum. That means mis-registration would appear. Figure 3 illustrates the conclusion clearly.
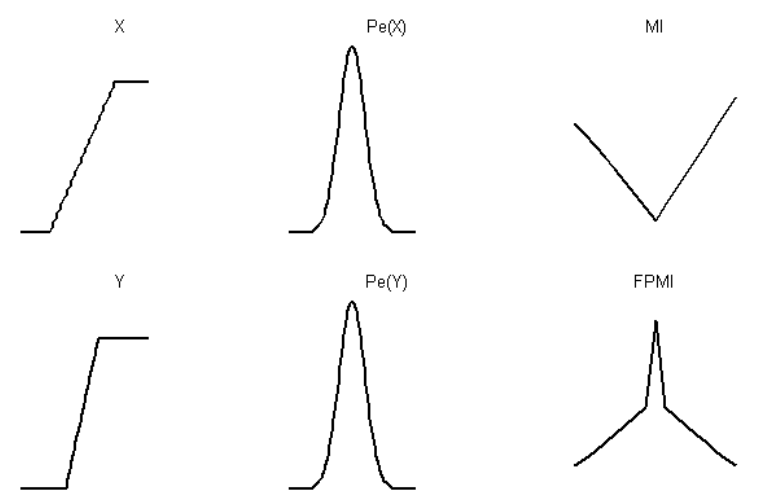

Figure 3: the first column is two edges with different edge widths. The second column is the feature potential corresponding left edge results. The up item of the third column is response of mutual information to horizon translation; the down item of the third column is the response of the FPMI to horizon translation.

\subsection{Registration of double edges}

When there are double edges in an image, location accuracy of double edge is dependent on the distance of two edges and the parameter of edge detection operator. In this case, registration accuracy of methods based on image segmentation is limited by the edge detection accuracy. However, FPMI could avoid this disadvantage greatly.

Suppose the double edges are $\mathrm{X}$ and $\mathrm{Y}$, the two feature potential function corresponding double edge are illustrated in figure 4. Because the $\sigma$ of potential function is so big that the two gauss apexes are merged to construct a potential apex in the middle of two edges. The feature potential function is sum by two gauss function, which is similar to a gauss function with big variance. The variance of the feature potential function is related to the distance of the double edges. 
Suppose the length of $\mathrm{X}$ and $\mathrm{Y}$ is $L$, the lengths of the merged gauss functions are $M$ and $N$ alternatively, and $N<M$. We transform signal $\mathrm{Y}$ along horizon direction. When signal $\mathrm{Y}$ shifts $x$ rightwards and $x \leq M$. $x<L-M$ is satisfied also. The FPMI is

$$
\begin{aligned}
F P M I_{x} & =-\frac{L-M-2 x}{2 L-2 x} \log \frac{L-M-2 x}{2 L-2 x}-\frac{L-M}{L-x} \log \frac{L-M}{2 L-2 x}-\frac{M-x}{L-x} \log \frac{1}{L-x} \\
& \approx-\log \frac{L-M}{2 L}-\left(1-\frac{L+M}{2 L-2 x}\right) \log \frac{L-M}{2 L}+\left(1-\frac{L-M}{L-x}\right) \log \frac{L-M}{2}
\end{aligned}
$$

Similar to the analysis of 3.1, FPMI $x$ is a monotonic decrease function with $x$. That means a maximum occurs at $x=0$. The conclusion is that for double edges with different distances, FPMI could reflect the registration degree of two double edges. Moreover, the distance of two edges does not influent the monotonic characteristic of FPMI, it only change $\sigma$ of potential function. Local maximum and mis-registration of FPMI would not appear long as the $\sigma$ is choose properly, no matter what distance between two edges is. That means the edge location accuracy would not influent the registration results greatly. This merit of FPMI is benefit to image registration.

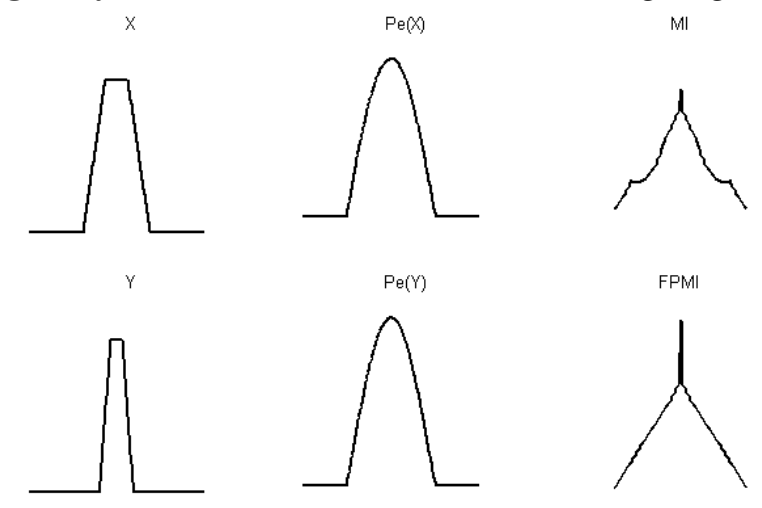

Figure 4: the first column is two double edges with different widths. The second column is the feature potential corresponding left edge results. The up item of the third column is response of mutual information to horizon translation; the down item of the third column is the response of the FPMI to horizon translation.

From above analysis we can see that for registration of single edge with same width, MI do registration well, FPMI do registration well also. For registration of single edge with different width, MI has local maxima, which results to misregistration. But FPMI has not local maxima and do registration well. For registration of double edges, registration accuracy of FPMI is well and edge detection accuracy would not influent registration results of FPMI greatly. That means the registration accuracy of FPMI is satisfied and robust.

\section{PARTICLE SWARM OPTIMIZATION}

Powell's method is a popular optimization method of mutual information in multimodality image registration. It repeatedly iterates the dimensions of the search space, performing one-dimensional optimizations for each dimension, until convergence is reached. However, this method could run into local maxima and reach local optimal results. In this paper, Particle Swarm Optimization (PSO) is applied to optimize the feature potential mutual information. To the authors' knowledge, PSO is rarely applied to registration. Wachowiak et.al ${ }^{[14]}$ proposed a new hybrid particle swarm technique that incorporates initial user guidance to register single-slice 3D to 3D biomedical images.

The PSO algorithm was first introduced by Eberhart and Kennedy ${ }^{[13]}$, it is another form of Evolutionary Computation and is stochastic in nature much like Genetic Algorithms. In a PSO system, particles fly around in a multidimensional search space. During flight, each particle adjusts its position according to its own experience, and according to the experience of a neighboring particle, making use of the best position encountered by itself and its neighbor. A minimization (or maximization) of the problem topology is found both by a particle remembering its own past best position and the companions' best overall position.

PSO is similar to GA in that the system is initialized with a population of random solutions. It combines local search methods with global search methods, attempting to balance exploration and exploitation. Its complex behavior follows from a few simple rules and has less computational complexity. 
In PSO, the $i$ th particle $x_{i}, i=1, \cdots, N$ moves by addition of a velocity vector $v_{i}$, which is a function of the best position found by the particle and of the best position found so far among all particles.

$$
\begin{aligned}
& v_{i}(k+1)=\phi(k) v_{i}(k)+\alpha_{1}\left[\gamma_{1 i}\left(p_{i}-x_{i}(k)\right)\right]+\alpha_{2}\left[\gamma_{2 i}\left(G-x_{i}(k)\right)\right] \\
& x_{i}(k+1)=x_{i}(k)+v_{i}(k+1)
\end{aligned}
$$

where, $i$ is particle index, $k$ is iteration index, $\phi(k)$ is the inertial weight. $v_{i}$ is the velocity of $i$ th particle, $x$ is the position of $i$ th particle, $p_{i}$ is the best position found by ith particle (personal best), $G$ is the best position found by swarm, $\gamma_{1,2}$ is the random numbers on the interval $[0,1]$ applied to $i$ th particle, $\alpha_{1,2}$ is acceleration constants.

In order to perform PSO, we set inertial weights $\phi(k)$ be monotonically decreasing function of the iterations index.

$$
\phi(k+1)=\phi(k)+\left(\frac{\phi_{\min }-\phi_{\max }}{K}\right)
$$

where $K$ is the iteration number. The iteration number is up to 200. $\phi_{\max }=0.9$ and $\phi_{\min }=0.4$. The population size is 20 and acceleration constants are $\alpha_{1}=\alpha_{2}=2$. Our search space is three dimensions, one rotation and two translations. $x_{i}=\left(t_{x}, t_{y}, \theta\right)$, where $t_{x}$ and $t_{y}$ are translation parameter, $\theta$ is rotation parameter.

\section{EXPERIMENTS}

(1) Interpolated images

In general, interpolators lead to smaller intensity errors in the interpolated image and result in interpolation artifacts of mutual information. We use interpolated MRI and CT images with different resolutions to compare the function of FPMI and standard MI. Let MRI be the reference image. CT image is translated along horizon direction and rotated alternatively. Spurious fluctuation in MI is caused by interpolation. However, FPMI function is smooth and has less fluctuation.
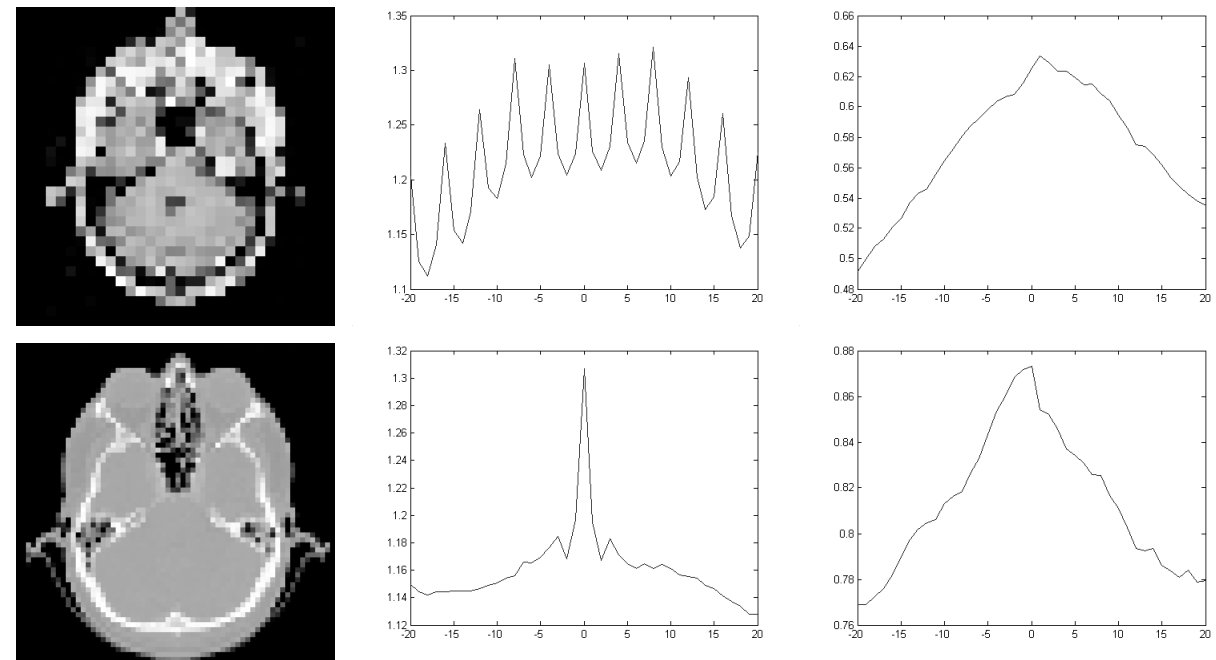

Figure 5: the first column is MRI images interpolated by nearest method. The second column is the responses of standard mutual information to horizon translation and rotation. The third column is the responses of FPMI to translation and rotation.

\section{(2) MRI-CBF}

The first column of figure 6 is MRI image and CBF image. They are registered already. Let MRI be the reference image and $\mathrm{CBF}$ be translated along vertical direction. Because the resolutions of these images are different, local fluctuations of standard mutual information occurs frequently. FPMI function is smoother and has less local fluctuations.

We transformed CT image according to known transformation parameters. Maximization of FPMI and standard MI using PSO is done to search the optimal registration parameters. In order to compare PSO and Powell's method, maximization of FPMI and standard MI using Powell's method is done also. Table 1 showed the registration results of 
maximization of FPMI and MI using PSO. Table 2 showed the registration results of maximization of FPMI and MI using Powell's method. We define registration error as,

$$
R E=\left|x-x^{*}\right|+\left|y-y^{*}\right|+\left|\theta-\theta^{*}\right|
$$

where $(x, y, \theta)$ is the registration result of horizon translation, vertical translation and rotation,$\left(x^{*}, y^{*}, \theta^{*}\right)$ is the known transformation parameter. Our registration accuracy is one pixel. From table 1 we can see that, no matter what optimization method used, the accuracy of the FPMI was more robust and more accurate than that of standard mutual information because of the spatial information.
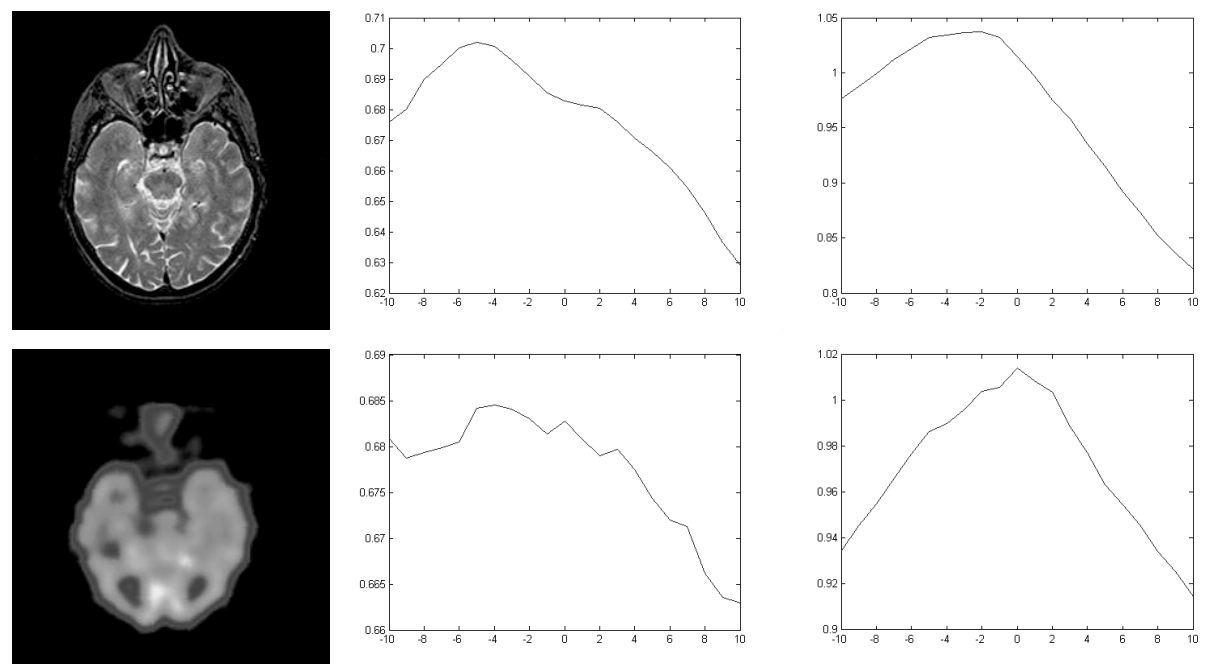

Figure 6: the first column is MRI and CBF images. The second column is the responses of standard mutual information to translation and rotation. The third column is the responses of feature potential mutual information to translation and rotation.

Table 1. Registration results of MRI-CBF by maximization of FPMI and MI using PSO

\begin{tabular}{|c|c|c|c|c|c|c|c|c|c|c|}
\hline \multicolumn{3}{|c|}{$\begin{array}{l}\text { Transformation } \\
\text { Parameters }\end{array}$} & \multicolumn{3}{|c|}{$\begin{array}{l}\text { Registration } \\
\text { results of our } \\
\text { method }\end{array}$} & \multicolumn{3}{|c|}{$\begin{array}{l}\text { Registration } \\
\text { results of MI }\end{array}$} & \multirow{2}{*}{$\begin{array}{c}\text { Registration } \\
\text { Error of our } \\
\text { method }\end{array}$} & \multirow{2}{*}{$\begin{array}{c}\text { Registration } \\
\text { Error of MI } \\
9\end{array}$} \\
\hline$(0$ & 4 & 7) & $(0$ & 6 & 7) & $(0$ & 9 & 11) & & \\
\hline$(-2$ & 0 & 5) & $(-2$ & 2 & 5) & $(-2$ & 5 & 8) & 2 & 8 \\
\hline$(5$ & 5 & 0) & $(5$ & 6 & 0) & ( 2 & 9 & 6) & 1 & 13 \\
\hline (5 & -3 & $-5)$ & $(5$ & 0 & $-5)$ & ( 3 & 2 & $-1)$ & 3 & 11 \\
\hline
\end{tabular}

Table 2. Registration results of MRI-CBF by maximization of FPMI and MI using Powell's method

\begin{tabular}{|c|c|c|c|c|c|c|c|c|c|c|}
\hline \multicolumn{3}{|c|}{$\begin{array}{c}\text { Transformation } \\
\text { Parameters }\end{array}$} & \multicolumn{3}{|c|}{$\begin{array}{l}\text { Registration results } \\
\text { of our method }\end{array}$} & \multicolumn{3}{|c|}{$\begin{array}{l}\text { Registration results } \\
\text { of MI }\end{array}$} & \multirow{2}{*}{$\begin{array}{c}\begin{array}{c}\text { Registration } \\
\text { Error of our } \\
\text { method }\end{array} \\
7\end{array}$} & \multirow{2}{*}{$\begin{array}{c}\text { Registration } \\
\text { Error of } \\
\text { MI }\end{array}$} \\
\hline$(0$ & 4 & 7 ) & $(1$ & 1 & 10) & $(-1$ & -1 & 10) & & \\
\hline$(-2$ & 0 & 5) & $(-1$ & -3 & 7) & $(-5$ & 6 & 3) & 6 & 11 \\
\hline ( 5 & 5 & 0) & ( 6 & 2 & $0)$ & ( 7 & 2 & 8) & 4 & 13 \\
\hline ( 5 & -3 & $-5)$ & ( 6 & -6 & $-6)$ & ( 6 & -8 & $-3)$ & 5 & 8 \\
\hline
\end{tabular}

\section{(3) MRI-CT}

The multimodal medical images in figure 7 are MRI image and CT image. They are registered already. The original image size is $256 \times 256$. Two images are downsampled to $32 \times 32$ to compute the response of FPMI and standard MI to vertical translation and rotation. Because the downsampled images are small, response of standard MI to translation and rotation could not reflect the registration degree between two images. It can be seen that although images are small, the 
FPMI function could illustrate the registration degree between two images. Moreover, the local maxima of FPMI are less than that of standard mutual information.

Table 3 showed the registration results of maximization of FPMI and MI using PSO. Table 4 showed the registration results of maximization of FPMI and MI using Powell's method. * means the search results is out of the search range. It can be seen that, PSO is better than Powell's method to search the optimal results when the local maxima of registration measure occur frequently. Whether PSO or Powell's method is done, registration results of FPMI are better than that of standard MI when standard MI could not reflect registration between two images.
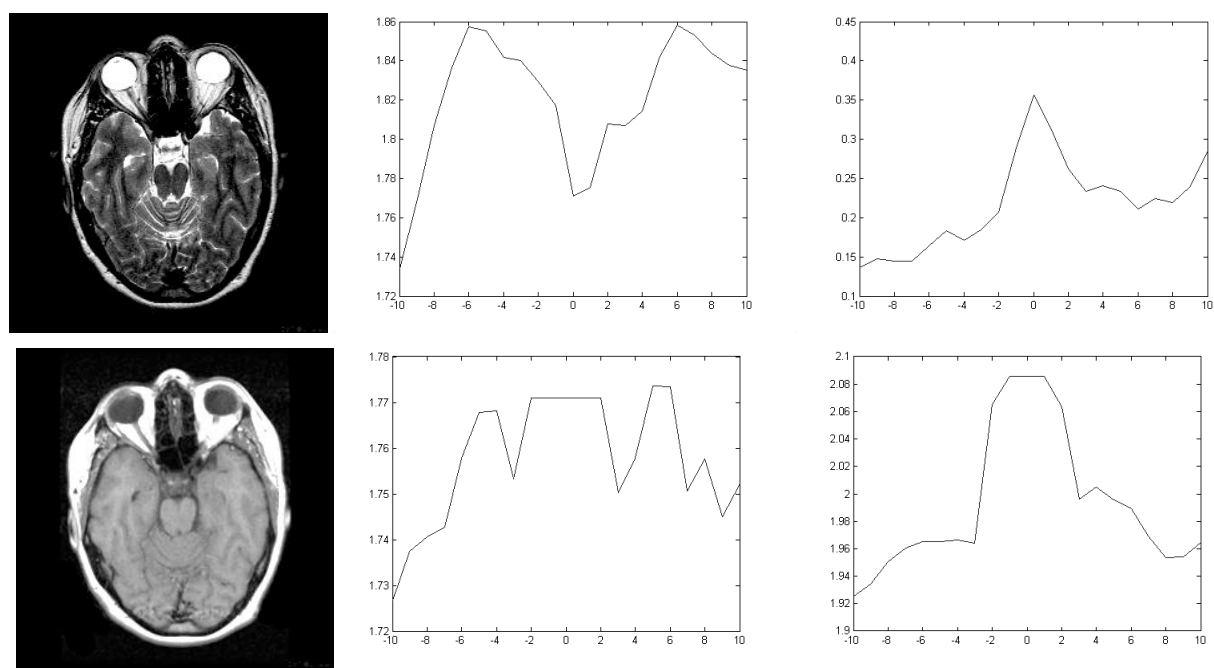

Figure 7: the first column is MRI and CT images. The second column is the responses of standard mutual information to vertical translation and rotation. The third column is the responses of FPMI to vertical translation and rotation.

Table 3. Registration results of MRI-CT using our method and maximization of standard Mutual Information

\begin{tabular}{|c|c|c|c|c|c|c|c|c|c|c|}
\hline \multicolumn{3}{|c|}{$\begin{array}{c}\text { Transformation } \\
\text { Parameters }\end{array}$} & \multicolumn{3}{|c|}{$\begin{array}{l}\text { Registration } \\
\text { results of our } \\
\text { method }\end{array}$} & \multicolumn{3}{|c|}{$\begin{array}{l}\text { Registration } \\
\text { results of MI }\end{array}$} & \multirow{2}{*}{$\begin{array}{c}\begin{array}{c}\text { Registration } \\
\text { Error of our } \\
\text { method }\end{array} \\
3\end{array}$} & \multirow{2}{*}{$\begin{array}{c}\begin{array}{c}\text { Registration } \\
\text { Error of } \\
\text { MI }\end{array} \\
8\end{array}$} \\
\hline$(0$ & 4 & 7) & $(0$ & 2 & 8) & $(0$ & 3 & 0) & & \\
\hline$(-2$ & 0 & 5) & $(-2$ & 0 & 8) & $(-2$ & 0 & 0) & 3 & 5 \\
\hline ( 5 & 4 & 0) & ( 4 & 1 & $-3)$ & ( 4 & 3 & 7) & 7 & 9 \\
\hline$(-5$ & 3 & 6) & $(-5$ & 2 & 9) & $(-5$ & 1 & 1) & 4 & 7 \\
\hline
\end{tabular}

Table 4. Registration results of MRI-CT using maximization of FPMI and MI by Powell's method

\begin{tabular}{|c|c|c|c|c|c|c|c|c|c|c|}
\hline \multicolumn{3}{|c|}{$\begin{array}{c}\text { Transformation } \\
\text { Parameters }\end{array}$} & \multicolumn{3}{|c|}{$\begin{array}{l}\text { Registration } \\
\text { results of FPMI }\end{array}$} & \multicolumn{3}{|c|}{$\begin{array}{l}\text { Registration } \\
\text { results of MI }\end{array}$} & \multirow{2}{*}{$\begin{array}{c}\text { Registration } \\
\text { Error of } \\
\text { FPMI }\end{array}$} & \multirow{2}{*}{$\begin{array}{l}\text { Registration } \\
\text { Error of MI } \\
16\end{array}$} \\
\hline$(0$ & 4 & 7) & $(-1$ & 4 & 9) & $(2$ & $12 *$ & 1) & & \\
\hline$(-2$ & 0 & 5) & $(-2$ & 1 & 4) & $(-8$ & $12 *$ & 7) & 2 & 20 \\
\hline ( 5 & 4 & 0) & $(5$ & 5 & $-3)$ & ( 6 & $12 *$ & $-1)$ & 4 & 10 \\
\hline$(-5$ & 3 & 6) & $(-5$ & 3 & 4) & $(-8$ & $12 *$ & 5) & 2 & 13 \\
\hline
\end{tabular}

\section{CONCLUSION}

Mutual information has developed into an accurate measure for multimodal medical image registration. But local extrema impede the registration optimization process and rule out the subpixel accuracy. We proposed the feature potential mutual information, which illustrate spatial information of images, to improve the registration of multimodality medical image. We detect edges of images and define feature potential using potential function. Feature 
potential mutual information is defined with feature potential of the two images. Particle Swarm Optimization is done to search the optimal transformation parameters. Theoretical analysis and experiments show that feature potential mutual information provides extremely accurate estimates of the alignment parameters. Moreover, in cases in which standard mutual information performs well, the functions of the FPMI are similar and the global optimum does not alter significantly. It is robust to interpolation noise and is feasible to register multimodal medical images. In the meantime, PSO is better than Powell's method to do optimization of registration measures.

\section{ACKNOWLEDGEMENTS}

This work is supported by China Guangdong Province Nature Science Found. Grant No. 31789.

\section{REFERENCES}

1. Josien P. W. Pluim, J. B. Antoine Maintz, and Max A. Viergever, "Mutual-Information-Based Registration of Medical Images: A Survey”, IEEE Trans Medical Imaging, 22, pp. 986-1004, 2003.

2. Jim Xiuquan Ji, Hao Pan, Zhi-Pei Liang, "Further Analysis of Interpolation Effects in Mutual Information-Based Image Registration", IEEE Trans. Medical Imaging, 22, pp. 1131-1140, 2003.

3. J.P.W. Pluim, J.B.A. Maintz and M.A. Viergever, "Interpolation artefacts in mutual information based image registration", Computer Vision and Image Understanding. 77, pp. 211-232, 2000.

4. Tsao, J., "Interpolation artifacts in multimodality image registration based on maximization of mutual information", IEEE Transactions on Medical Imaging, 22, pp. 854 - 864, 2003.

5. J.P.W. Pluim, J.B.A. Maintz and M.A. Viergever, "Image registration by maximization of combined mutual information and gradient information", IEEE Transactions on Medical Imaging, 19, pp. 809-814, 2000.

6. P. Viola and W. M. Wells III, Alignment by Maximization of Mutual Information, Proceedings of the 5th International Conference on Computer Vision, pp.16-23, Cambridge, MA, 1995.

7. A.Collignon, F. Maes, D. Delaere, D. Vandermeulen, P. Suetens, and G. Marchal, "Automated multi-modality image registration based on information theory", Information Processing in Medical Imaging, pp. 263-274, The Netherlands: Kluwer, 1995.

8. B.Likar, F. Pernus, "A hierarchical approach to elastic registration based on mutual information", Image and Vision Computing, 19, pp. 33-44, 2001.

9. M. B. Skouson, Quji Guo, Z. P. Liang, “A Bound on Mutual Information for Image Registration”, IEEE Trans. Medical Imaging, 20, pp. 843-846, 2001.

10. Raj Shekhar and Vladimir Zagrodsky, "Mutual Information-Based Rigid and Nonrigid Registration of Ultrasound Volumes", IEEE Trans. Medical Imaging, 21, pp. 9-22, 2002.

11. C. Studholme, D. L. G. Hill, and D. J. Hawkes, "An overlap invariant entropy measure of 3D medical image alignment”, Pattern Recognit., 32, pp. 71-86, 1999.

12. F. Maes, A. Collignon, D. Vandermeulen, G. Marchal, and P. Suetens, "Multimodality image registration by maximization of mutual information", IEEE Trans. Med. Imag., 16, pp. 187-198, 1997.

13. Eberhart, R.C., and Kennedy, J., "A new optimizer using particle swarm theory", Proc. Sixth International Symposium on Micro Machine and Human Science, IEEE Service Center, pp. 39-43, Nagoya, Japan, 1995.

14. M.P.Wachowiak, R.Smoliková, Yufeng Zheng, J.M.Zurada, A.S.Elmaghraby, "An Approach to Multimodal Biomedical Image Registration Utilizing Particle Swarm Optimization”, IEEE Trans. Evolutionary Computation, 8, pp. 289-301, 2004. 